\title{
Stochastic Stability Analysis for Markovian Jump Neutral Nonlinear Systems
}

\author{
Bo Wang ${ }^{1}$ Peng Shi ${ }^{2}$ Hamid Reza Karimi ${ }^{3}$ Xiucheng Dong ${ }^{1}$
}

\author{
${ }^{1}$ School of Electrical and Information Engineering, Xihua University, Chengdu, 610096, China. E-mail: \\ bowang@uestc.edu.cn \\ School of Applied Mathematics, University Electronic Science and Technology of China, Chengdu 610054, China. \\ ${ }^{2}$ School of Electrical and Electronic Engineering, The University of Adelaide, Adelaide, SA 5005, Australia. E- \\ mail: peng.shi@adelaide.edu.au \\ School of Engineering and Science, Victoria University, Melbourne, Vic 8001, Australia. E-mail: \\ Peng.Shi@vu.edu.au \\ ${ }^{3}$ Department of Engineering, Faculty of Engineering and Science, University of Agder, N-4898 Grimstad, Norway. \\ Corresponding Author: E-mail: hamid.r.karimi@uia.no
}

\begin{abstract}
In this paper, the stability problem is studied for a class of Markovian jump neutral nonlinear systems with time-varying delay. By Lyapunov-Krasovskii function approach, a novel mean-square exponential stability criterion is derived for the situations that the systems transition rates are completely accessible, partially accessible and non-accessible, respectively. Moreover, the developed stability criterion is extended to the systems with different bounded sector nonlinear constraints. Finally, some numerical examples are provided to illustrate the effectiveness of the proposed methods.
\end{abstract}

Keywords: Stochastic stability; Markovian jump system, nonlinear system, time-varying delay

\section{Introduction}

Among stochastic hybrid systems, a widely studied class is the so-called Markovian jump systems (MJSs), which are described by a set of systems with commutation generated by a finite state Markov chain. It has been shown that MJSs have great application potentials, such as biomedicine, fault tolerant systems, networked control, economics, aerospace, and communication networks. Due to the probabilistic description of commutations, MJSs are very suitable and effective to describe dynamical systems with the model changes induced by external causes, such as unexpected events, random faults, uncontrolled configuration changes. A great amount of effort has been devoted to MJSs in the past years, and many useful and important results have been reported in literature. In Ma et al. (2012), a stochastic version of a bounded real lemma was derived for a general class of discrete-time time-varying MJSs with state- and disturbance-multiplicative noise, and the necessary and sufficient conditions for the solvability of the control problem in terms of four coupled discrete-time Riccati equations are presented. In Zhang (2009), the estimation problem was studied for a class of MJSs with time-varying transition probabilities, which was considered to be finite piecewise homogeneous. In Costa and Oliveira (2012), the stochastic optimal control problem was researched for discretetime linear systems subject to Markov jumps and multiplicative noises under two criteria. In Fei et al. (2009), a new delay-dependent stochastic stability criterion for a class of MJSs with time delay was derived based on a novel Lyapunov-Krasovskii functional ap- 
proach. In Wu et al. (2012), the problem on the sliding mode control of Markov jump singular time-delay systems is concerned through investigation of the bounded gain performance in the analysis of sliding mode dynamics in order to improve the system transient performance. Recently in Karimi (2011), an efficient approach is developed for robust $H_{\infty}$ control problem of uncertain time-delay systems with Markovian switching parameters and mixed discrete, neutral and distributed delays. For more state-of-the-art results on this topic, readers are referred to (Karimi (2012), Wu et al. (2011), Basin and Shi (2009), Shi and Liu (2011), Wu et al. (2010), Liu et al. (2011b)) and the references therein.

In real systems, time delays exist widely. Hence recently much effort has been included in such area, see Cai et al. (2012), Liu et al. (2011a) and references therein. Meanwhile,the transition rates of MJSs are, in general, not completely accessible. Hence the problems of modeling, analysis and design on MJSs with partially transition rates have become an important and challenging issue, and attracted a lot of attentions, see for example, Shi et al. (2012), Yin et al. (2012), Luan et al. (2010), Zhang et al. (2009), Zhang et al. (2011), Zhang et al. (2009), Wang et al. (2010) and the references therein. However, most theoretical results either have some strict limitations with regard to transition rates matrix, such as main diagonal elements are required, or have some conservatism for not fully utilizing the provided transition rates. In addition, for MJSs with time-varying delay, most stability criteria have the constraints that the derivative of time-varying delay must be less than a constant, such as 1 , which limits the applied scope. In this paper, all the available commutation information will be utilized to derive a stochastic stability criterion, independent of time-varying delays derivative, for Markovian jump neutral nonlinear delay systems (MJNNSs), no matter if transition rates are completely accessible, partially accessible and non-accessible. Moreover, the developed stability criterion will be extended to systems with different bounded sector nonlinear constraints. Some numerical examples will be included to demonstrate the effectiveness of the obtained theoretical results.

The remainder of this paper is organized as follows. The MJNNS with time-varying delay and some preliminaries are provided in Section 2. Section 3 presents the results on stochastic stability analysis. Section 4 gives an illustrative example, and the paper is concluded in Section 5. Notations used in this paper are fairly standard. Let $R^{n}$ be the n-dimensional Euclidean space, $R^{n \times m}$ represents the set of $n \times m$ real matrices, the symbol * denotes the elements below the main diagonal of a symmetric block matrix, $A>0$ means that
$A$ is a real symmetric positive definitive matrix, $I$ denotes the identity matrix with appropriate dimensions. $\operatorname{diag}\{\cdots\}$ denotes the diagonal matrix. $\mathrm{E}\{\cdot\}$ refers to the expectation operator with respect to some probability measure P. $\|\cdot\|$ refers to the Euclidean vector norm or the induced matrix 2-norm. The superscript T stands for matrix transposition, $L_{n, h}=L\left([-h, 0], R^{n}\right)$ denotes the Banach space of continuous functions mapping the interval $[-h, 0]$ into $R^{n}$ with the topology of uniform convergence.

\section{Model description and preliminaries}

In this paper, $(\Omega, \Upsilon, P)$ denotes the probability space, where $\Omega$ is the sample space, $\Upsilon$ is $\sigma$ - algebra of subset of the sample space, and $\mathrm{P}$ is the probability measure defined on $\Upsilon$. Let $S=\{1,2, \ldots, N\}$ and the random form process $\{r(t), t \in[0,+\infty)\}$ be a homogeneous, finite state Markov process with right continuous trajectories with generator $\Pi=\left(\pi_{i j}\right)_{N \times N}$ and transition probability form mode $i$ at time $t$ to mode $j$ at time $t+\Delta t, i \in S, j \in S$, given by

$$
\begin{aligned}
& P\{r(t+\Delta t)=j \mid r(t)=i\} \\
& =\left\{\begin{array}{l}
\pi_{i j} \Delta t+o(\Delta t), j \neq i \\
1+\pi_{i i} \Delta t+o(\Delta t), j=i
\end{array}\right.
\end{aligned}
$$

where transition rates $\pi_{i j} \geq 0, j \neq I, \pi_{i i}=$ $-\sum_{j=1, j \neq i}^{N} \pi_{i j}, \Delta t>0$, and $\lim _{\Delta t \rightarrow 0} o(\Delta t) / \Delta t=0$.

For the convenience of study, the transition rates of the Markov chain are considered to be partially accessible. Namely, some elements in transition rates matrix are time invariant but unknown, such as a system with three operation modes may have the transition rates matrix as follow

$$
\Pi=\left[\begin{array}{ccc}
- & \pi_{12} & \pi_{13} \\
\pi_{21} & \pi_{22} & \pi_{23} \\
- & \pi_{32} & -
\end{array}\right]<0
$$

where - represents the inaccessible element.

Throughout the paper, we denote $S=S_{k n}^{i} \cup S_{u n}^{i}$ for $\forall i \in S$, where $S_{k n}^{i}=\left\{j: \pi_{i j}\right.$ is known $\}$ and $S_{u k}^{i}=$ $\left\{j: \pi_{i j}\right.$ is unknown $\}$. First, consider the following MJNNS

$$
\left\{\begin{array}{l}
\dot{x}(t)=A(r(t)) x(t)+B(r(t)) x(t-\tau(t)) \\
+C(r(t)) \dot{x}(t-d)+D(r(t)) f(t, z(t)) \\
z(t)=L x(t)+N x(t-\tau(t)) \\
x\left(t_{0}+\theta\right)=\psi(\theta), \theta \in[-h, 0]
\end{array}\right.
$$

where $x(t) \in R^{n}$ is the state vector, $0 \leq d \leq h$ is the neutral delay, $0 \leq \tau(t) \leq h$ is the time-varying 
delay, $A(r(t)) \in R^{n \times n}, B(r(t)) \in R^{n \times n}, C(r(t)) \in$ $R^{n \times n}, D(r(t)) \in R^{n \times n}, L \in R^{m \times n}, N \in R^{m \times n}$ are matrix functions of the random jumping process $\{r(t)\}$. When $r(t)=i \in S$, the matrices $A(r(t)), B(r(t)), C(r(t)), D(r(t))$ are represented by $A_{i}, B_{i}, C_{i}, D_{i}, \psi(\theta) \in L_{n, h}$ is the initial condition, $f(t) \in R^{n}$ is the nonlinear term, and satisfies the following Lipchitz condition

$$
f^{T}(t, z(t))(f(t, z(t))-K z(t)) \leq 0
$$

We have

$$
\begin{aligned}
& -2 f^{T}(t, z(t)) f(t, z(t))+2 f^{T}(t, z(t)) K(L x(t) \\
& +N x(t-\tau(t))) \leq 0
\end{aligned}
$$

Then, model (1) can be represented as

$$
\left\{\begin{array}{l}
\dot{x}(t)=y(t) \\
y(t)=A(r(t)) x(t)+B(r(t)) x(t-\tau(t)) \\
+C(r(t)) y(t-d)+D(r(t)) f(t, z(t)) \\
z(t)=L x(t)+N x(t-\tau(t))
\end{array}\right.
$$

Remark 1: It is shown in Bao et al. (2009) that the $L\left([-h, 0], R^{n}\right) \times S$ - valued process $\left(x_{t}, r(t)\right)$ is a time homogenous strong Markov process, where $x_{t}=$ $\{x(t+\theta):-h \leq \theta \leq 0\}$.

Now, to present the main objective of this paper more precisely, we also introduce the following definition for system (1), which is essential for the later development.

Definition 1: System (1) is mean-square exponentially stable if there exist scalars $\alpha>0$ and $\beta>0$ such that

$$
\mathrm{E}\|x(t)\|^{2} \leq \alpha e^{-\beta t}\|\psi\|_{h}^{2}
$$

where $\|\psi\|_{h}=\max \left\{\sup _{h \leq \theta \leq 0}\|\psi(\theta)\|\right\}$.

Therefore, in this paper, the authors attention will be focused on the development of mean-square exponential stability criterion independent of time-varying delays derivative for the situations that the systems transition rates are completely accessible, partially accessible and non-accessible, respectively.

Before ending the section, we give the following lemmas, which will be used in the proof of our main results.

Lemma 1 (Boyd et al. (1994)): For any constant matrices E, G and $\mathrm{F}$ with appropriate dimensions, $F^{T} F \leq k I, \mathrm{k}$ is a positive scalar, then

$$
2 x^{T} E F G y \leq c x E E^{T} x+\frac{k}{c} y^{T} G^{T} G y
$$

where $\mathrm{c}$ is a positive scalar, $x \in R^{n}$, and $y \in R^{n}$.

Lemma 2 (Gu (2000)): For any positive definite matrix $\Phi \in R^{n \times n}$, scalar $\gamma>0$, vector function $w$ :
$[0, \gamma] \rightarrow R^{n}$ such that the integrations concerned are well defined, then

$$
\left(\int_{0}^{\gamma} w(s) d s\right)^{T} \Phi\left(\int_{0}^{\gamma} w(s) d s\right) \leq \gamma \int_{0}^{\gamma} w^{T}(s) \Phi w(s) d s
$$

\section{Main results}

In this section, based on Lyapunov method and linear matrix inequality techniques, the following stochastic stability criteria are derived.

Theorem 1: When $\pi_{i i}$ is unknown with given $h$ and $k$, if there exist $P_{2}, P_{3}, U, V, W$, and symmetric positive-definite matrices $F_{i}, R, M, S, Q$, such that

$$
\begin{gathered}
{\left[\begin{array}{cc}
\left(\sum_{j \in S_{k n}^{i}} \pi_{i j}\right) T_{i} & T_{i 1}^{k n} \\
* & T_{i 2}^{k n}
\end{array}\right]<0} \\
{\left[\begin{array}{cc}
T_{i} & T_{i 1 j}^{u k} \\
* & T_{i 2 j}^{u k}
\end{array}\right]<0, \forall j \neq i, j \in S_{u k}^{i}} \\
{\left[\begin{array}{cc}
W & U \\
* & S-R_{22}
\end{array}\right]>0} \\
{\left[\begin{array}{cc}
W & V \\
* & S
\end{array}\right]>0}
\end{gathered}
$$

where

$$
\Sigma_{i}=\left[\begin{array}{cccccc}
\Sigma_{1,1} & \Sigma_{1,2} & \Sigma_{1,3} & \Sigma_{1,4} & \Sigma_{1,5} & \Sigma_{1,6} \\
* & \Sigma_{2,2} & \Sigma_{2,3} & \Sigma_{2,4} & \Sigma_{2,5} & \Sigma_{2,6} \\
* & * & \Sigma_{3,3} & 0 & 0 & 0 \\
* & * & * & \Sigma_{4,4} & 0 & 0 \\
* & * & * & * & \Sigma_{5,5} & 0 \\
* & * & * & * & * & \Sigma_{6,6}
\end{array}\right]
$$

$$
\begin{aligned}
& \Sigma_{1,1}=P_{2} A_{i}+A_{i}^{T} P_{2}^{T}+Q+k F_{i} \\
& \Sigma_{1,2}=F_{i}-P_{2}+A_{i}^{T} P_{3}^{T}, \Sigma_{1,3}=P_{2} B_{i}+R_{12}{ }^{T} \\
& \Sigma_{2,2}=h e^{k h} S-P_{3}-P_{3}^{T}+M, \Sigma_{2,3}=P_{3} B_{i} \\
& \Sigma_{3,3}=h R_{11}-R_{12}-R_{12}{ }^{T}, \Sigma_{1,4}=P_{2} C_{i} \\
& \Sigma_{2,4}=P_{3} C_{i}, \Sigma_{4,4}=-M e^{-k h} \\
& \Sigma_{1,5}=P_{2} D_{i}+L^{T} K^{T}, \Sigma_{2,5}=P_{3} D_{i} \\
& \Sigma_{3,5}=N^{T} K^{T}, \Sigma_{5,5}=-2 I, \Sigma_{6,6}=-Q e^{-k h} \\
& T_{i}=\Sigma_{i}+\Omega+\Omega^{T}+h e^{k h} W \\
& T_{i 1}^{k n}=\left[\sum_{j \in S_{k n}^{i}} \pi_{i j} F_{j}, 0,0,0,0,0\right]^{T} \\
& T_{i 2}^{k n}=-\sum_{j \in S_{k n}^{i}} \pi_{i j} F_{j}, T_{i 1 j}^{u k}=\left[F_{j}, 0,0,0,0,0\right]^{T} \\
& T_{i 2 j}^{u k}=-F_{j}, \Omega=[U, 0,-U+V, 0,0,-V]
\end{aligned}
$$

then, MJNNS (1) is mean-square exponentially stable. 
Proof: Choose the following Lyapunov-Krasovskii functional candidate

$$
V(t, r(t))=\sum_{j=1}^{6} V_{j}(t, r(t))
$$

where

$$
\begin{aligned}
& V_{1}(t, r(t))=\left(\begin{array}{ll}
x^{T}(t) & \left.y^{T}(t)\right)
\end{array}\left[\begin{array}{ll}
I & 0 \\
0 & 0
\end{array}\right]\right. \\
& \times\left[\begin{array}{cc}
F_{i}(r(t)) & 0 \\
P_{2}^{T} & P_{3}^{T}
\end{array}\right]\left(\begin{array}{ll}
x^{T}(t) & \left.y^{T}(t)\right)^{T}
\end{array}\right. \\
& V_{2}(t, r(t))=\int_{-h}^{0} \int_{t+\beta}^{t} y^{T}(\alpha) e^{k(\alpha-t+h)} S y(\alpha) d \alpha d \beta \\
& \left.V_{3}(t, r(t))\right)=\int_{-h}^{t} \int_{\beta-\tau(\beta)}^{\beta} \eta^{T} e^{k(\beta-t)} R \eta d \alpha d \beta \\
& \left.V_{4}(t, r(t))\right)=\int_{t-d}^{t} y^{T}(s) e^{k(s-t)} M y(s) d s \\
& \left.V_{5}(t, r(t))\right)=\int_{-h}^{0} \int_{t+\beta}^{t} \xi^{T}(\alpha) e^{k(\alpha-t+h)} W \xi(\alpha) d \alpha d \beta \\
& \left.V_{6}(t, r(t))\right)=\int_{t-h}^{t} x^{T}(s) e^{k(s-t)} Q x(s) d s
\end{aligned}
$$

with

$$
\begin{aligned}
& \eta=\left[x^{T}(\beta-\tau(\beta)), y^{T}(\alpha)\right]^{T} \\
& \xi(t)=\left[\begin{array}{l}
x^{T}(t), y^{T}(t), x^{T}(t-\tau(t)), \\
y^{T}(t-d), f^{T}(t), x^{T}(t-h)
\end{array}\right]^{T}
\end{aligned}
$$

Let $L$ be the infinitesimal generator of random process. Then for each $r(t)=i, i \in S$, we have

$$
L V(t, i)=\sum_{j=1}^{6} L V_{j}(t, i)
$$

where

$$
\begin{aligned}
& L V_{1}(t, i) \leq 2 x^{T}(t) P_{2} A_{i} x(t)+2 x^{T}(t) P_{2} D_{i} f(t) \\
& +2 x^{T}(t)\left(F_{i}-P_{2}+A^{T}{ }_{i} P_{3}{ }^{T}\right) y(t)-2 y^{T}(t) P_{3} y(t) \\
& +2 x^{T}(t) P_{2} C_{i} y(t-d)+2 x^{T}(t) P_{2} B_{i} x(t-\tau(t)) \\
& +2 y^{T}(t) P_{3} B_{i} x(t-\tau(t))+2 y^{T}(t) P_{3} C_{i} y(t-d) \\
& +2 y^{T}(t) P_{3} D_{i} f(t)+x^{T}(t)\left(\sum_{j \neq i, j=1}^{N} \pi_{i j} F_{j}\right) x(t)
\end{aligned}
$$

$L V_{2}(t, i) \leq h y^{T}(t) e^{k h} S y(t)-\int_{t-\tau(t)}^{t} y^{T}(s) S y(s) d s$ $-\int_{t-h}^{t-\tau(t)} y^{T}(s) S y(s) d s-k V_{2}(t, i)$

$$
\begin{aligned}
& L V_{3}(t, i) \leq h x^{T}(t-\tau(t)) R_{11} x(t-\tau(t)) \\
& +2 x^{T}(t) R_{12}{ }^{T} x(t-\tau(t))+\int_{t-\tau(t)}^{t} y(s) R_{22} y(s) d s \\
& -2 x^{T}(t-\tau(t)) R_{12} x(t-\tau(t))-k V_{3}(t, i)
\end{aligned}
$$$$
L V_{4}(t, i) \leq y^{T}(t) M y(t)-y^{T}(t-d) e^{-k h} M y(t-d)
$$$$
-k V_{4}(t, i)
$$$$
L V_{5}(t, i) \leq h \xi^{T}(t) e^{k h} W \xi(t)-\int_{t-\tau(t)}^{t} \xi^{T}(s) W \xi(s) d s
$$$$
-\int_{t-h}^{t-\tau(t)} \xi^{T}(s) W \xi(s) d s-k V_{5}(t, i)
$$

$$
\begin{aligned}
& L V_{6}(t, i)=x^{T}(t) Q x(t)-x^{T}(t-h) e^{-k h} Q x(t-h) \\
& -k V_{6}(t, i)
\end{aligned}
$$

Hence, we get

$$
\begin{aligned}
& L V(t, i) \leq \xi^{T}(t) \Xi_{i} \xi(t)-\int_{t-\tau(t)}^{t} q^{T}(s) \Phi_{1} q(s) d s \\
& -\int_{t-h}^{t-\tau(t)} q^{T}(s) \Phi_{2} q(s) d s-k V(t, i)
\end{aligned}
$$

where

$$
\begin{aligned}
& \Xi_{i}=T_{i}+\operatorname{diag}\left\{\Psi_{i}, 0,0,0,0,0\right\} \\
& \Psi_{i}=\sum_{j \neq i, j \in S} \pi_{i j} F_{j}, q(t)=\left[\xi^{T}(s), y^{T}(s)\right]^{T} \\
& \Phi_{1}=\left[\begin{array}{cc}
W & U \\
* & S-R_{22}
\end{array}\right], \Phi_{2}=\left[\begin{array}{cc}
W & V \\
* & S
\end{array}\right]
\end{aligned}
$$

From (7) and (8), we know that $L V(t, i) \leq-k V(t, i)$ hold if $\Xi_{i}<0$.

Consider $\Psi_{i} \geq 0$ and $1 \geq \sum_{i \neq j, j \in S^{i}} \pi_{i j} \geq 0$, we can derive that $L V(t, i) \leq-k V(t, i)$ holds if

$$
\left(\sum_{i \neq j, j \in S^{i}} \pi_{i j}\right) T_{i}+\operatorname{diag}\left\{\Psi_{i}, 0,0,0,0,0\right\}<0
$$

By Schur complement, the above inequality can be rewritten as

$$
\begin{aligned}
& {\left[\begin{array}{cc}
\left(\sum_{j \in S_{k n}^{i}} \pi_{i j}\right) T_{i} & T_{i 1}^{2 k n} \\
* & T_{i 2}^{2 k n}
\end{array}\right]} \\
& +\sum_{j \neq i, j \in S_{u k}^{i}} \pi_{i j}\left[\begin{array}{cc}
T_{i} & T_{i 1 j}^{2 u k} \\
* & T_{i 2 j}^{2 u k}
\end{array}\right]<0
\end{aligned}
$$

With ineq.(5) - ineq.(8), we can conclude that

$$
L V(t, i) \leq-k V(t, i)
$$

Hence

$$
L\left(e^{k t} V(t, i)\right)=e^{k t}(L V(t, i)+k V(t, i)) \leq 0
$$

By Dynkins formula, one can obtain

$$
\begin{aligned}
& \mathrm{E} V(t, i) e^{k t}=\mathrm{E} V\left(t_{0}, i\right) e^{k t_{0}}+\mathrm{E} \int_{t_{0}}^{t} L\left(e^{k s} V(s, i)\right) d s \\
& \leq \mathrm{E} V\left(t_{0}, i\right) e^{k t_{0}}
\end{aligned}
$$

Hence

$$
\lambda_{\min }\left(F_{i}\right) \mathrm{E}\|x(t)\|^{2} \leq \mathrm{E} V(t, i) \leq \mathrm{E} V\left(t_{0}, i\right) e^{-k\left(t-t_{0}\right)}
$$

From the definition of $V(t, i)$, we know that

$$
\begin{aligned}
& \mathrm{E} V\left(t_{0}, i\right) \leq\left[\lambda_{\max }\left(F_{i}\right)+h^{2} \lambda_{\max }(S)+h^{2} \lambda_{\max }(R)\right. \\
& \left.+h \lambda_{\max }(M)+h^{2} \lambda_{\max }(W)+h \lambda_{\max }(Q)\right] \mathrm{E}\|\psi\|_{h}{ }^{2}
\end{aligned}
$$


where $\|\psi\|_{h}=\sup _{-h \leq \theta \leq 0}\|\psi(\theta)\|$.

Then, the following inequality is deduced

$$
\mathrm{E}\|x(t)\| \leq a e^{-\frac{k}{2}\left(t-t_{0}\right)} \mathrm{E}\|\psi\|_{h}
$$

where

$$
a=\sqrt{\frac{\left(\begin{array}{l}
\lambda_{\max }\left(F_{i}\right)+h^{2} \lambda_{\max }(S)+h^{2} \lambda_{\max }(R) \\
+h \lambda_{\max }(M)+h^{2} \lambda_{\max }(W)+h \lambda_{\max }(Q)
\end{array}\right)}{\lambda_{\min }\left(F_{i}\right)}}
$$

Finally, with Definition 1, we can conclude that MJNNS (1) is mean-square exponentially stable. The proof of Theorem 1 is thus completed.

Theorem 1 investigated MJNNS (1) in the situation that $\pi_{i i}$ is unknown. Based on Theorem 1, the following theoretical result can be derived.

Corollary 1: When $\pi_{i i}$ is known with given $h$ and $k$, if there exist $P_{2}, P_{3}, U, V, W$, and symmetric positivedefinite matrices $F_{i}, R, M, S, Q$, such that

$$
\begin{aligned}
& {\left[\left(1+\sum_{j \in S_{k n}^{i}} \pi_{i j}\right) \bar{T}_{i} \quad \bar{T}_{i 1}^{k n}\right]<0} \\
& {\left[\begin{array}{cc}
\bar{T}_{i} & \bar{T}_{i 1 j}^{u k} \\
* & \bar{T}_{i 2 j}^{u k}
\end{array}\right]<0, \forall j \in S_{u k}^{i}} \\
& {\left[\begin{array}{cc}
W & U \\
* & S-R_{22}
\end{array}\right]>0} \\
& {\left[\begin{array}{ll}
W & V \\
* & S
\end{array}\right]>0}
\end{aligned}
$$

where

$$
\bar{\Sigma}_{i}=\left[\begin{array}{cccccc}
\Sigma_{1,1} & \Sigma_{1,2} & \Sigma_{1,3} & \Sigma_{1,4} & \Sigma_{1,5} & \Sigma_{1,6} \\
* & \Sigma_{2,2} & \Sigma_{2,3} & \Sigma_{2,4} & \Sigma_{2,5} & \Sigma_{2,6} \\
* & * & \Sigma_{3,3} & 0 & 0 & 0 \\
* & * & * & \Sigma_{4,4} & 0 & 0 \\
* & * & * & * & \Sigma_{5,5} & 0 \\
* & * & * & * & * & \Sigma_{6,6}
\end{array}\right]
$$

$$
\begin{aligned}
& \Sigma_{1,1}=P_{2} A_{i}+A_{i}^{T} P_{2}^{T}+\pi_{i i} F+Q_{i}+k F_{i} \\
& \Sigma_{1,2}=F_{i}-P_{2}+A_{i}^{T} P_{3}^{T} \\
& \Sigma_{2,2}=h e^{k h} S-P_{3}-P_{3}^{T}+M \\
& \Sigma_{1,3}=P_{2} B_{i}+R_{12}{ }^{T}, \Sigma_{2,3}=P_{3} B_{i} \\
& \Sigma_{3,3}=h R_{11}-R_{12}-R_{12}{ }^{T}, \Sigma_{1,4}=P_{2} C_{i} \\
& \Sigma_{2,4}=P_{3} C_{i}, \Sigma_{4,4}=-M e^{-k h} \\
& \Sigma_{1,5}=P_{2} D_{i}+L^{T} K^{T}, \Sigma_{2,5}=P_{3} D_{i} \\
& \Sigma_{3,5}=N^{T} K^{T}, \Sigma_{5,5}=-2 I, \Sigma_{6,6}=-Q e^{-k h} \\
& \bar{T}_{i}=\bar{\Sigma}_{i}+\Omega+\Omega^{T}+h e^{k h} W \\
& \bar{T}_{i 1}^{k n}=\left[\sum_{j \neq i, j \in S_{k n}^{i}} \pi_{i j} F_{j}, 0,0,0,0,0\right]^{T} \\
& \bar{T}_{i 2}^{k n}=-\sum_{j \neq i, j \in S_{k n}^{i}} \pi_{i j} F_{j}, \bar{T}_{i 1 j}^{u k}=\left[F_{j}, 0,0,0,0,0\right]^{T} \\
& \bar{T}_{i 2 j}^{u k}=-F_{j}, \Omega=[U, 0,-U+V, 0,0,-V]
\end{aligned}
$$

then, MJNNS (1) is mean-square exponentially stable. Next, we consider the MJNNS with following nonlinear constraints

$$
\left(f(t, z(t))-K_{1} z(t)\right)^{T}\left(f(t, z(t))-K_{2} z(t)\right) \leq 0
$$

where $K_{2}>K_{1}$.

Let $F(t, z(t))=f(t, z(t))-K_{1} z(t)$, we have

$$
F^{T}(t, z(t))(F(t, z(t))-K z(t)) \leq 0
$$

where $K=K_{2}-K_{1}$.

The following inequality can be obtained

$$
\begin{aligned}
& -2 F^{T}(t, z(t)) F(t, z(t))+2 F^{T}(t, z(t)) K(L x(t) \\
& +N x(t-\tau(t))) \geq 0
\end{aligned}
$$

Then MJNNS (1) can be represented as follows

$$
\left\{\begin{array}{l}
\dot{x}(t)=\bar{A}(r(t)) x(t)+\bar{B}(r(t)) x(t-\tau(t)) \\
+C(r(t)) \dot{x}(t-d)+D(r(t)) F(t, z(t)) \\
z(t)=L x(t)+N x(t-\tau(t)) \\
x\left(t_{0}+\theta\right)=\psi(\theta), \theta \in[-h, 0]
\end{array}\right.
$$

where $\bar{A}(r(t))=A(r(t))+D(r(t)) K_{1} L, \bar{B}(r(t))=$ $B(r(t))+D(r(t)) K_{1} N$.

It can be seen that system (1) and system (27) have the same structure with different system parameters. Hence based on Theorem 1, the following theoretical result can be derived.

Theorem 2: When $\pi_{i i}$ is unknown with given $h$ and $k$, if there exist $P_{2}, P_{3}, U, V, W$, and symmetric positive-definite matrices $F_{i}, R, M, S, Q$, such that

$$
\begin{gathered}
{\left[\begin{array}{cc}
\left(\sum_{j \in S_{k n}^{i}} \pi_{i j}\right) T_{i} & T_{i 1}^{k n} \\
* & T_{i 2}^{k n}
\end{array}\right]<0} \\
{\left[\begin{array}{cc}
T_{i} & T_{i 1 j}^{u k} \\
* & T_{i 2 j}^{u k}
\end{array}\right]<0, \forall j \neq i, j \in S_{u k}^{i}} \\
{\left[\begin{array}{cc}
W & U \\
* & S-R_{22}
\end{array}\right]>0} \\
{\left[\begin{array}{cc}
W & V \\
* & S
\end{array}\right]>0}
\end{gathered}
$$

where

$$
\Sigma_{i}=\left[\begin{array}{cccccc}
\Sigma_{1,1} & \Sigma_{1,2} & \Sigma_{1,3} & \Sigma_{1,4} & \Sigma_{1,5} & \Sigma_{1,6} \\
* & \Sigma_{2,2} & \Sigma_{2,3} & \Sigma_{2,4} & \Sigma_{2,5} & \Sigma_{2,6} \\
* & * & \Sigma_{3,3} & 0 & 0 & 0 \\
* & * & * & \Sigma_{4,4} & 0 & 0 \\
* & * & * & * & \Sigma_{5,5} & 0 \\
* & * & * & * & * & \Sigma_{6,6}
\end{array}\right]
$$




$$
\begin{aligned}
& \Sigma_{1,1}=P_{2}\left(A_{i}+D_{i} K_{1} L\right)+\left(A_{i}+D_{i} K_{1} L\right)^{T} P_{2}^{T} \\
& +Q+k F_{i}, \Sigma_{1,2}=F_{i}-P_{2}+\left(A_{i}+D_{i} K_{1} L\right)^{T} P_{3}{ }^{T} \\
& \Sigma_{2,2}=h e^{k h} S-P_{3}-P_{3}^{T}+M, \Sigma_{2,4}=P_{3} C_{i} \\
& \Sigma_{1,3}=P_{2}\left(B_{i}+D_{i} K_{1} N\right)+R_{12} T \\
& \Sigma_{2,3}=P_{3}\left(B_{i}+D_{i} K_{1} N\right), \Sigma_{1,4}=P_{2} C_{i} \\
& \Sigma_{3,3}=h R_{11}-R_{12}-R_{12},_{2,5}=P_{3} D_{i} \\
& \Sigma_{1,5}=P_{2} D_{i}+L^{T} K^{T}, \Sigma_{3,5}=N^{T} K^{T} \\
& \Sigma_{4,4}=-M e^{-k h}, \Sigma_{5,5}=-2 I, \Sigma_{6,6}=-Q e^{-k h} \\
& T_{i}=\Sigma_{i}+\Omega+\Omega^{T}+h e^{k h} W \\
& T_{i 1}^{k n}=\left[\sum_{j \in S_{k n}^{i}} \pi_{i j} F_{j}, 0,0,0,0,0\right]^{T} \\
& T_{i 2}^{k n}=-\sum_{j \in S_{k n}^{i}} \pi_{i j} F_{j}, T_{i 1 j}^{u k}=\left[F_{j}, 0,0,0,0,0\right]^{T} \\
& T_{i 2 j}^{u k}=-F_{j}, \Omega=[U, 0,-U+V, 0,0,-V]
\end{aligned}
$$

then, MJNNS (27) is mean-square exponentially stable.

Proof: The proof is similar to that of Theorem 1 , and thus is omitted.

Theorem 2 studied MJNNS (27) in the situation that $\pi_{i i}$ is unknown. Based on Theorem 2, the following theoretical result can be derived.

Corollary 2: When $\pi_{i i}$ is known with given $h$ and $k$, if there exist $P_{2}, P_{3}, U, V, W$, and symmetric positivedefinite matrices $F_{i}, R, M, S, Q$, such that

$$
\begin{gathered}
{\left[\begin{array}{cc}
\left(1+\sum_{j \in S_{k n}^{i}} \pi_{i j}\right) \bar{T}_{i} & \bar{T}_{i 1}^{k n} \\
* & \bar{T}_{i 2}^{k n}
\end{array}\right]<0} \\
{\left[\begin{array}{cc}
\bar{T}_{i} & \bar{T}_{i 1 j}^{u k} \\
* & \bar{T}_{i 2 j}^{u k}
\end{array}\right]<0, \forall j \in S_{u k}^{i}} \\
{\left[\begin{array}{cc}
W & U \\
* & S-R_{22}
\end{array}\right]>0} \\
{\left[\begin{array}{cc}
W & V \\
* & S
\end{array}\right]>0}
\end{gathered}
$$

where

$$
\bar{\Sigma}_{i}=\left[\begin{array}{cccccc}
\Sigma_{1,1} & \Sigma_{1,2} & \Sigma_{1,3} & \Sigma_{1,4} & \Sigma_{1,5} & \Sigma_{1,6} \\
* & \Sigma_{2,2} & \Sigma_{2,3} & \Sigma_{2,4} & \Sigma_{2,5} & \Sigma_{2,6} \\
* & * & \Sigma_{3,3} & 0 & 0 & 0 \\
* & * & * & \Sigma_{4,4} & 0 & 0 \\
* & * & * & * & \Sigma_{5,5} & 0 \\
* & * & * & * & * & \Sigma_{6,6}
\end{array}\right]
$$

$$
\begin{aligned}
& \Sigma_{1,1}=P_{2}\left(A_{i}+D_{i} K_{1} L\right)+\left(A_{i}+D_{i} K_{1} L\right)^{T} P_{2}^{T} \\
& +\pi_{i i} F_{i}+Q+k F_{i}, \Sigma_{2,3}=P_{3}\left(B_{i}+D_{i} K_{1} N\right) \\
& \Sigma_{1,2}=F_{i}-P_{2}+\left(A_{i}+D_{i} K_{1} L\right)^{T} P_{3}^{T} \\
& \Sigma_{2,2}=h e^{k h} S-P_{3}-P_{3}^{T}+M, \Sigma_{1,4}=P_{2} C_{i} \\
& \Sigma_{1,3}=P_{2}\left(B_{i}+D_{i} K_{1} N\right)+R_{12},^{T}, \Sigma_{2,4}=P_{3} C_{i} \\
& \Sigma_{3,3}=h R_{11}-R_{12}-R_{12} T, \Sigma_{4,4}=-M e^{-k h} \\
& \Sigma_{1,5}=P_{2} D_{i}+L^{T} K^{T}, \Sigma_{2,5}=P_{3} D_{i} \\
& \Sigma_{3,5}=N^{T} K^{T}, \Sigma_{5,5}=-2 I, \Sigma_{6,6}=-Q e^{-k h} \\
& \bar{T}_{i}=\bar{\Sigma}_{i}+\Omega+\Omega^{T}+h e^{k h} W \\
& \bar{T}_{i 1}^{k n}=\left[\sum_{j \neq i, j \in S_{k n}^{i}} \pi_{i j} F_{j}, 0,0,0,0,0\right]^{T} \\
& \bar{T}_{i 2}^{k n}=-\sum_{j \neq i, j \in S_{k n}^{i}} \pi_{i j} F_{j}, \bar{T}_{i 1 j}^{u k}=\left[F_{j}, 0,0,0,0,0\right]^{T} \\
& \bar{T}_{i 2 j}^{u k}=-F_{j}, \Omega=[U, 0,-U+V, 0,0,-V]
\end{aligned}
$$

then, MJNNS (27) is mean-square exponentially stable.

\section{Simulation Results}

In this section, we will verify the proposed methodology by giving an illustrative example. The MJNNS with following system parameters is included

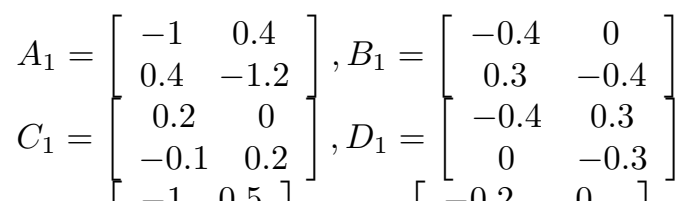

$$
\begin{aligned}
& A_{2}=\left[\begin{array}{ll}
-1 & 0.5 \\
0.4 & -1
\end{array}\right], B_{2}=\left[\begin{array}{cc}
-0.2 & 0 \\
0.3 & -0.3
\end{array}\right] \\
& C_{2}=\left[\begin{array}{cc}
0.1 & 0 \\
-0.1 & 0.1
\end{array}\right], D_{2}=\left[\begin{array}{cc}
-0.3 & 0.2 \\
0 & -0.4
\end{array}\right] \\
& A_{3}=\left[\begin{array}{ll}
-1 & 0.4 \\
0.3 & -1
\end{array}\right], B_{3}=\left[\begin{array}{cc}
-0.5 & 0.1 \\
0.4 & -0.3
\end{array}\right] \\
& C_{3}=\left[\begin{array}{cc}
0.2 & 0.1 \\
-0.2 & -0.1
\end{array}\right], D_{3}=\left[\begin{array}{cc}
-0.4 & 0.1 \\
0.1 & -0.2
\end{array}\right] \\
& L=\left[\begin{array}{cc}
0.2 & 0 \\
0 & 0.2
\end{array}\right], N=\left[\begin{array}{cc}
0.3 & 0 \\
0 & 0.3
\end{array}\right] \\
& \tau(t)=0.6+0.1 \sin ^{2}(15 t), k=0.1, d=0.4 \\
& f(t, z(t))=(|z(t)+1|-|z(t)-1|) / 2, K=I
\end{aligned}
$$

First we consider the given MJNNS with transition rates as follow

$$
\Pi_{1}=\left[\begin{array}{ccc}
-0.5 & 0.3 & 0.2 \\
0.3 & -0.9 & 0.6 \\
0.2 & 0.2 & -0.4 \\
-0.7 & - & - \\
0.4 & -0.5 & 0.1 \\
0.1 & 0.3 & -0.4 \\
-0.5 & - & - \\
- & -0.3 & - \\
0.4 & 0.2 & -0.6
\end{array}\right]
$$

According to the derived stability criteria, Table 1 can be obtained. 
Table 1: The maximum stable delay of the MJNNS with different transition rates

\begin{tabular}{cccc}
\hline transition rates & case 1 & case 2 & case 3 \\
\hline$h$ & 1.1434 & 1.0093 & 0.9225 \\
\hline
\end{tabular}

Next, we consider the given MJNNS with transition rates that are unknown, partially known and completely known as follow

$$
\begin{aligned}
& \Pi_{4}=\left[\begin{array}{ccc}
- & - & - \\
- & - & - \\
- & - & -
\end{array}\right] \\
& \Pi_{5}=\left[\begin{array}{ccc}
- & - & - \\
0.2 & -0.4 & 0.2 \\
0.2 & 0.3 & -0.5
\end{array}\right] \\
& \Pi_{6}=\left[\begin{array}{ccc}
-0.6 & 0.3 & 0.3 \\
0.2 & -0.4 & 0.2 \\
0.2 & 0.3 & -0.5
\end{array}\right]
\end{aligned}
$$

According to the presented theoretical results, Table 2 can be obtained.

Table 2: The maximum stable delay of the MJNNS with transition rates unknown, partly known and fully known

\begin{tabular}{cccc}
\hline trans. rates & unknown & par. known & ful. known \\
\hline$h$ & 0.7507 & 0.8149 & 1.1524 \\
\hline
\end{tabular}

Remark 2: Table 1 shows the maximum stable delay of the MJNNS changes with different transition rates. This means that transition rates have an effect on the stochastic stability of the MJNNS. Table 2 displays the relationship between the maximum stable delay of the MJNNS and transition rates that are unknown, partially known and fully known, respectively. It can be seen that the biggest maximum stable delay occurs in the situation that transition rates are fully known for the best utilization of all commutation information. In addition, it is illustrated that no matter how many transition rates are accessible, the presented stability criteria are still available.

Next, the numerical simulation is carried out to analyze the dynamics of the MJNNS. The numerical simulation is with the initial state value $\psi(\theta)=$ $[1,-1.2]^{T}, \theta \in(-1,0), r\left(t_{0}\right)=2$, the simulation step 0.001 second, the transition rates $\Pi_{6}$ and the switching time 0.1 second. Corresponding simulation results are shown in Figs. 1 and 2.

Remark 3: Fig. 1 depicts the time response of the state variable of the MJNNS. Fig. 2 depicts the time
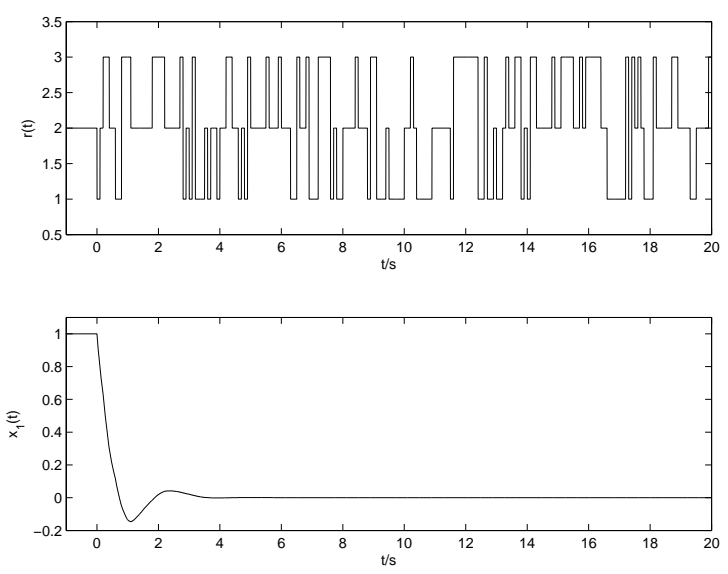

Figure 1: Time response of the state variable $x_{1}(t)$ of the MJNNS.

response of the state variable of the MJNNS. It can be seen that the MJNNS is mean-square exponential stable, and spends about 6 seconds converging to the zero state.

Remark 4: For the given example, $\dot{\tau}(t)=$ $1.5 \sin (30 t)$, and the maximum upper bound of $\dot{\tau}(t)$ is 1.5 , greater than 1. Based on the constructed Lyapunov function, the derived stochastic stability criteria have no constrains on the upper bound of $\dot{\tau}(t)$. In addition, the calculated maximum stable delay of the MJNNS is 1.1524, bigger than the real maximum delays upper bound 0.7 , which means that this system should be mean-square exponentially stable, and the simulation results in Figs. 1 and 2 accord with the proposed stability criteria.
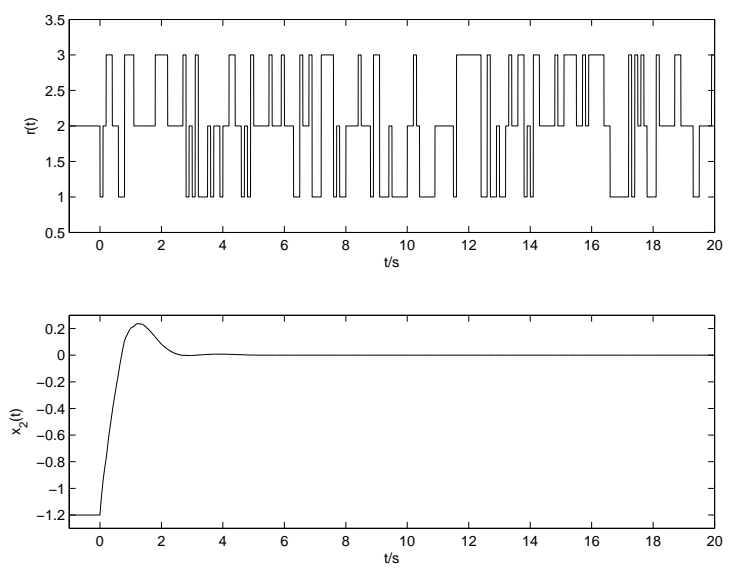

Figure 2: Time response of the state variable $x_{2}(t)$ of the MJNNS. 


\section{Conclusions}

In this paper, the stochastic stability analysis on Markovian jump neutral nonlinear systems with timevarying delay is concerned. By constructing a novel Lyapunov function, the mean-square exponential stability criterion, independent of time-varying delays derivative, has been concluded for the circumstance that transition rates are completely accessible, partially accessible and non-accessible. Subsequently, the developed stability criterion has been extended to the systems with a different bounded sector nonlinear constrain. At last, some numerical examples have been included to illustrate the effectiveness of the obtained theoretical results.

\section{Acknowledgements}

This work was partially supported by the Key Projects of Xihua University (Z1120946), the National Key Basic Research Program (973), China (No. 2012CB215202), the 111 Project (B12018), the National Natural Science Foundation of China (Nos. 61174058, 61170030), and the Engineering and Physical Sciences Research Council, UK (EP/F029195).

\section{References}

Bao, J., Houa, Z., and Yuan, C. Stability in distribution of neutral stochastic differential delay equations with Markovian switching. Statistics and Probability Letters, 2009. 79:1663-1673. doi:10.1016/j.spl.2009.04.006.

Basin, M. and Shi, P. Guest Editorial: New trends in optimal and robust filtering for stochastic systems. Circuits Systems and Signal Processing, 2009. 28:185-189. doi:10.1007/s00034-008-9076-1.

Boyd, S., Feron, L. G. E., and Balakrishnan, V. Linear matrix inequalities in system and control theory. Philadelphia, PA, SIAM, 1994.

Cai, G., Hu, C., and Duan, G. Efficient LMI-Based Quadratic Stability and Stabilization of ParameterDependent Interval Systems with Applications. International Journal of Innovative Computing, Information and Control, 2012. 8:1943-1954.

Costa, O. and Oliveira, A. Optimal mean - variance control for discrete-time linear systems with Markov jumps and multiplicative noises. Automatica, 2012. 48:304-315. doi:10.1016/j.automatica.2011.11.009.
Fei, Z., Gao, H., and Shi, P. New results on stabilization of Markov jump systems with time delay. Automatica, 2009. 45:2300-2306. doi:10.1016/j.automatica.2009.06.020.

$\mathrm{Gu}, \mathrm{K}$. An integral inequality in the stability problem of time-delay systems. The 39th IEEE Conference on Decision Control, Sydney, Australia, 2000. pages 2805-2810. doi:10.1109/CDC.2000.914233.

Karimi, H. R. Robust delay-dependent $H_{\infty}$ control of uncertain Markovian jump systems with mixed neutral, discrete and distributed time-delays. IEEE Trans. Circuits and Systems I, 2011. 58:1910-1923. doi:10.1109/TCSI.2011.2106090.

Karimi, H. R. A sliding mode approach to $H_{\infty}$ synchronization of master-slave time-delay systems with Markovian jumping parameters and nonlinear uncertainties. Journal of the Franklin Institute, 2012. 349:480-1496. doi:10.1016/j.jfranklin.2011.09.015.

Liu, J., Gu, Z., and Hu, S. $H_{\infty}$ Filtering for Markovian Jump Systems with Time-Varying Delays. International Journal of Innovative Computing, Information and Control, 2011a. 7:1299-1310.

Liu, M., Shi, P., Zhang, L., and Zhao, X. Fault tolerant control for nonlinear Markovian jump systems via proportional and derivative sliding mode observer. IEEE Trans on Circuits and Systems I: Regular Papers, 2011b. 58:2755-2764. doi:10.1109/TCSI.2011.2157734.

Luan, X., Liu, F., and Shi, P. Finite-time filtering for non-linear stochastic systems with partially known transition jump rates. IET Control Theory and Applications, 2010. 4:735-745. doi:0.1049/ietcta.2009.0014.

Ma, H., Zhang, W., and Hou, T. Infinite horizon $H_{2} / H_{\infty}$ control for discrete-time timevarying Markov jump systems with multiplicative noise. Automatica, 2012. 48:1447-1454. doi:10.1016/j.automatica.2012.05.006.

Shi, P. and Liu, M. Discussion on "On the filtering problem for continuous-time Markov jump linear systems with no observation of the Markov chain". European J. of Control, 2011. 17:355-356. doi:10.1007/s00034-008-9076-1.

Shi, P., Luan, X., and Liu, F. $H_{\infty}$ filtering for discrete-time systems with stochastic incomplete measurement and mixed delays. IEEE Trans on Industrial Electronics, 2012. 59:2732-2739. doi:10.1109/TIE.2011.2167894. 
Wang, G., Zhang, Q., and Sreeram, V. Partially mode-dependent filtering for discrete-time Markovian jump systems with partly unknown transition probabilities. Signal Processing, 2010. 90:548-556. doi:10.1016/j.sigpro.2009.07.020.

Wu, L., Shi, P., and Gao, H. State estimation and sliding mode control of Markovian jump singular systems. IEEE Trans on Automatic Control, 2010. 55:1213-1219. doi:10.1109/TAC.2010.2042234.

Wu, L., Su, X., and Shi, P. Sliding mode control with bounded gain performance of Markov jump singular time-delay systems. Automatica, 2012. 48:19291933. doi:10.1016/j.automatica.2012.05.064.

Wu, Z., Shi, P., Su, H., and Chu, J. $L_{2}-$ $L_{\infty}$ filter design for discrete-time singular Markovian jump systems with time-varying delays. Information Sciences, 2011. 181:5534-5534. doi:10.1016/j.ins.2011.07.052.
Yin, Y., Shi, P., Liu, F., and Pan, J. Gain-scheduled fault detection on stochastic nonlinear systems with partially known transition jump rates. Nonlinear Analysis: Real World Applications, 2012. 13:359369. doi:10.1016/j.nonrwa.2011.07.043.

Zhang, L. $H_{\infty}$ estimation for discrete-time piecewise homogeneous Markov jump linear systems. Automatica, 2009. 45:2570-2576. doi:10.1016/j.automatica.2009.07.004.

Zhang, L., Boukas, K., and Shi, P. $H_{\infty}$ model reduction for discrete-Time Markov Jump linear systems with partially known transition. Int. J. of Control, 2009. 82:243-351. doi:10.1080/00207170802098899.

Zhang, L., Boukas, K., and Shi, P. Gain scheduled PI tracking control on stochastic nonlinear systems with partially known transition probabilities. J. of Franklin Institute, 2011. 348:685-702. doi:10.1016/j.jfranklin.2011.01.011. 